\title{
Visibilizando a las cirujanas en Chile
}

\author{
Claudia R. Albornoz G. ${ }^{1}$, Katya Carrillo G. ${ }^{2}$, Victoria Vargas V. ${ }^{3}$, Pamela Pedrero B. ${ }^{4}$, \\ Macarena Fernández ${ }^{5}$, Verónica Fonseca S. ${ }^{6}$, Lorena Pérez S. ${ }^{7}$, Carolina Rodríguez P. , \\ Karen Rojas R. ${ }^{9}$, Ingrid Plass D. ${ }^{4}$ y Jamile Camacho N. ${ }^{10}$
}

${ }^{1}$ Hospital Clínico Universidad de Chile Clínica Las Condes. ${ }^{2}$ Hospital de La Florida, Hospital Clínico Universidad de Chile.

Hospital de Punta Arenas. ${ }^{4}$ Instituto Nacional del Cánce ${ }^{5}$ Hospital San Borja Arriarán.

${ }^{6}$ Hospital de Temuco,

Universidad de la Frontera.

7Hospital de Talca, Universidad

Católica del Maule.

'Hospital de Curicó.

${ }^{9}$ Hospital de Coquimbo.

${ }^{10}$ Clínica Alemana de Santiago.

Recibido 2020-07-27 y aceptado 2020-09-29.

Correspondencia a:

Dra. Claudia R. Albornoz clau.albornoz@gmail.com

\section{Making female surgeons visible in Chile}

Introduction: Surgery traditionally has been considered a male discipline. It is unknown if the increase in the number of female doctors in the last decades has increased the number of female surgeons. Aim: Is to analyze and make visible the historical and current participation of women in Medicine and Surgery in Chile. Materials and Method: Retrospective cohort study of all medical doctors and surgeons graduated from chilean Universities since 1970 to date, and cross-sectional study to know current information of female surgeons and residents. Source of data were the Registries of Health Superintendence, CONACEM and the Registry of the Female Surgeon Association. Results: There has been a steady increase in the number of graduated female doctors in Chile, surpassing male doctors since 2018. Female surgeons are $15 \%$ of all surgeons, and the number has duplicated every decade since 1970s. Currently, 33\% of the residents are female. Main area of developing is general surgery (35\%), and within subspecialties: Breast, Plastics, and Head and Neck. Conclusions: Women are still underrepresented in Surgery: however, there has been a steady increase and that trend is expected to continue. It is necessary to make visible the importance of female surgeons to be able to increase female representation.

Key words: surgeon; gender; gender equity.

\section{Resumen}

Introducción: La cirugía ha sido tradicionalmente considerada una especialidad masculina. Se desconoce si el aumento en el número de médicas en las últimas décadas ha producido un aumento significativo en el número de cirujanas. Objetivo: Analizar y visibilizar la participación actual e histórica de las mujeres en Medicina y en Cirugía General en Chile. Materiales y Método: Estudio retrospectivo de la cohorte de médicas/os y cirujanas/os egresados de escuelas de medicina chilenas desde el año 1970, complementado con estudio de corte transversal para conocer la información actual de las cirujanas y residentes. Las fuentes de datos fueron los Registros de la Superintendencia de Salud, CONACEM y el Catastro de la Asociación de Cirujanas. Resultados: Ha existido un aumento sostenido de médicas egresadas sobrepasando a sus pares masculinos a partir de 2018. Las cirujanas representan el $15 \%$ del total de cirujanas y cirujanos, y este número se ha duplicado por década a partir de los años 70 . Actualmente, $33 \%$ de los residentes en formación son de género femenino. Las áreas más comunes de desarrollo son cirugía general (35\%), y dentro de las subespecialidades: mama, plástica y cabeza y cuello. Conclusión: Las mujeres siguen siendo minoría en cirugía; sin embargo, se ha producido un aumento progresivo y se espera siga la misma tendencia. Es necesario visibilizar la importancia de las cirujanas para que sirvan como modelo a nuevas generaciones de estudiantes y así poder aumentar la representación femenina en la especialidad.

Palabras clave: cirujanas; género; equidad de género.

\section{Introducción}

La cirugía ha sido tradicionalmente considerada un campo masculino; sin embargo, las mujeres han tenido un rol importante en medicina y cirugía des- de tiempos antiguos ${ }^{1}$. Documentos demuestran la existencia de mujeres cirujanas en el antiguo Egipto, Mesopotamia y Roma. El Tetrabiblion, libro que describió las técnicas quirúrgicas de Aspasia, cirujana grecorromana, fue escrito en el año 150 a.C, y 
fue utilizado como texto de estudio hasta el siglo XI. Sin embargo, el ejercicio de la cirugía fue prohibido para las mujeres en la Edad Media. En la Edad Moderna, para poder practicar la cirugía, las mujeres debieron "camuflarse" de hombre; conocidos son los casos de Elena/Eleno de Céspedes (1545-1588), cirujana española que aprendió y practicó exitosamente la cirugía impersonada de hombre, y el de James Barry (1795-1865), cirujano escocés que tuvo una importante participación en el ejército inglés y que en el momento de su muerte se descubrió que había nacido y vivido como mujer (Margaret Ann Bulkley) hasta la edad en que se enroló en el ejército y comenzó a ejercer la cirugía ${ }^{2}$.

Afortunadamente, los tiempos han cambiado y las mujeres no tienen que vestirse como hombre para poder ser cirujanas. Sin embargo, las barreras para las mujeres en cirugía siguen existiendo. Actualmente, más del $50 \%$ de los estudiantes que ingresan a estudiar Medicina son mujeres, sin embargo, un porcentaje muy pequeño elige cirugía como especialidad ${ }^{1}$. En un estudio de Espinoza et al. de 2012, 8,4\% de la fuerza quirúrgica en Chile correspondía a mujeres ${ }^{3}$.

En Chile se ha producido una incorporación progresiva de la mujer al trabajo, $52,7 \%$ de las mujeres participan en el mundo laboral comparado con $73,8 \%$ de los hombres ${ }^{4}$. En las carreras de la Salud, entre los años 2007 y 2018 en nuestro país, las egresadas de género femenino duplicaron a sus pares masculinos ${ }^{5}$. Sin embargo, no hay información acerca de la participación de las mujeres en Medicina y específicamente, en Cirugía. El objetivo principal de esta investigación es analizar y visibilizar la participación actual e histórica de las mujeres en Medicina y en Cirugía en Chile, y describir las características sociodemográficas de las cirujanas y el área en que se desarrollan.

\section{Materiales y Método}

Se realizó un estudio descriptivo retrospectivo de la cohorte histórica de cirujanas certificadas en los últimos 50 años en Chile (1970 a la fecha), y se complementó con un estudio de corte transversal para identificar la información actual de las cirujanas. Se analizó:

1. Porcentaje actual de médicos de género femenino y masculino en Chile, y su evolución histórica.

2. Porcentaje actual de cirujanos de género femenino y masculino en Chile, y su evolución histórica.

3. Porcentaje actual de cirujanos de género femenino por subespecialidad.
4. Edad de las cirujanas y lugar de ejercicio de la profesión.

5. Lugar de formación.

6. Pertenencia a la Sociedad de Cirujanos de Chile.

7. Porcentaje actual de cirujanas en formación.

Se obtuvo estadísticas descriptivas: porcentajes, promedios, medianas, rangos. El estudio siguió las directrices del código de Nuremberg para estudios con humanos.

Los criterios de inclusión en el estudio fueron:

1. Médicos/as cirujanos/as certificados en la Superintendencia de Salud por universidades chilenas o por la Corporación Nacional Autónoma de Certificación de Especialidades Médicas (CONACEM).

2. Especialidad de Cirugía General certificada por universidades chilenas o CONACEM. Se eliminaron datos duplicados: por ejemplo, si un médico/a se encontraba certificado por una universidad y CONACEM, se contabilizó como certificado por universidad.

\section{Fuentes de información y análisis}

Para el cálculo del porcentaje de médicas en Chile, porcentaje de cirujanas, evolución del porcentaje de médicas, cirujanas y lugar de formación, la fuente de información fue el Registro de Prestadores Individuales (RNPI) de la Superintendencia de Salud a la fecha de 30 de abril de $2020^{6}$. El RNPI es la base de datos más fidedigna y completa de los profesionales de la salud del país, informa la habilitación legal de los profesionales de la salud para ejercer sus respectivas profesiones, en virtud de ostentar sus respectivos títulos profesionales, así como sobre la certificación de las especialidades médicas.

Los porcentajes de médicas se obtuvieron dividiendo el número de médicas por el total de médicos (hombres y mujeres) y se multiplicó por 100. Los porcentajes de cirujanas total se obtuvieron dividiendo el número de cirujanas por el total de cirujanos (hombres y mujeres) y se multiplicó por 100 .

Para el análisis de la evolución histórica del porcentaje de cirujanas certificadas en Chile, se calculó el porcentaje de cirujanas certificadas por década en comparación con el total de ambos géneros. Es posible que la certificación por CONACEM no coincida exactamente con el término de la formación.

El porcentaje actual de cirujanas por subespecialidad se obtuvo del RNPI para las subespecialidades de cirugía cardíaca, vascular, plástica, cabeza y cuello y coloproctología. Los datos de la subespecialidad de cirugía digestiva fueron obtenidos 


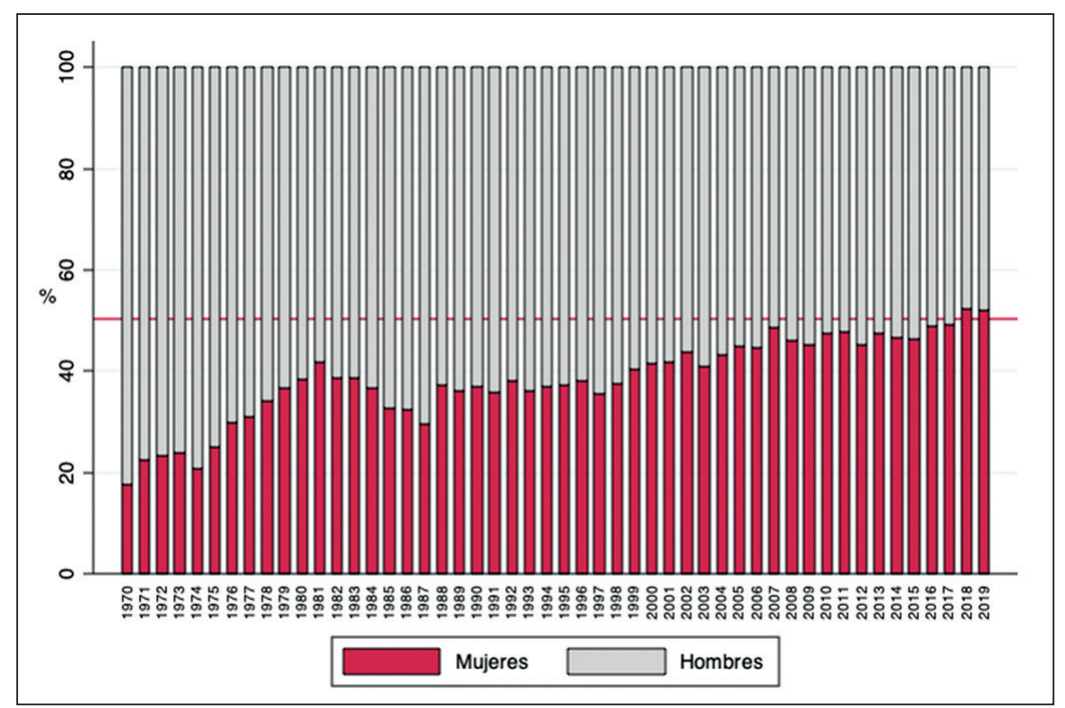

Figura 1. Proporción histórica de mujeres egresadas de escuelas de Medicina en Chile 19702019. Fuente: Superintendencia de Salud.

\section{Participación de mujeres en Medicina}

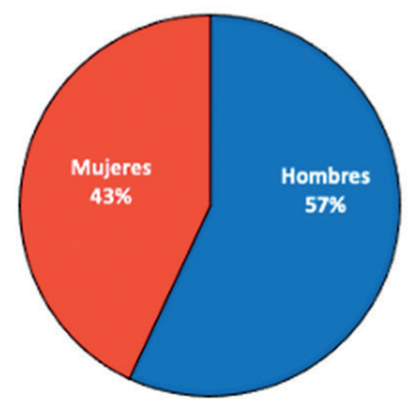

\section{Participación de mujeres en Cirugía}

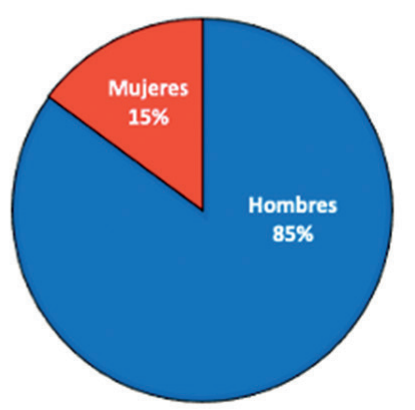

Figura 2. Participación de mujeres en Medicina y en Cirugía en Chile, respectivamente. Fuente: Superintendencia de Salud.

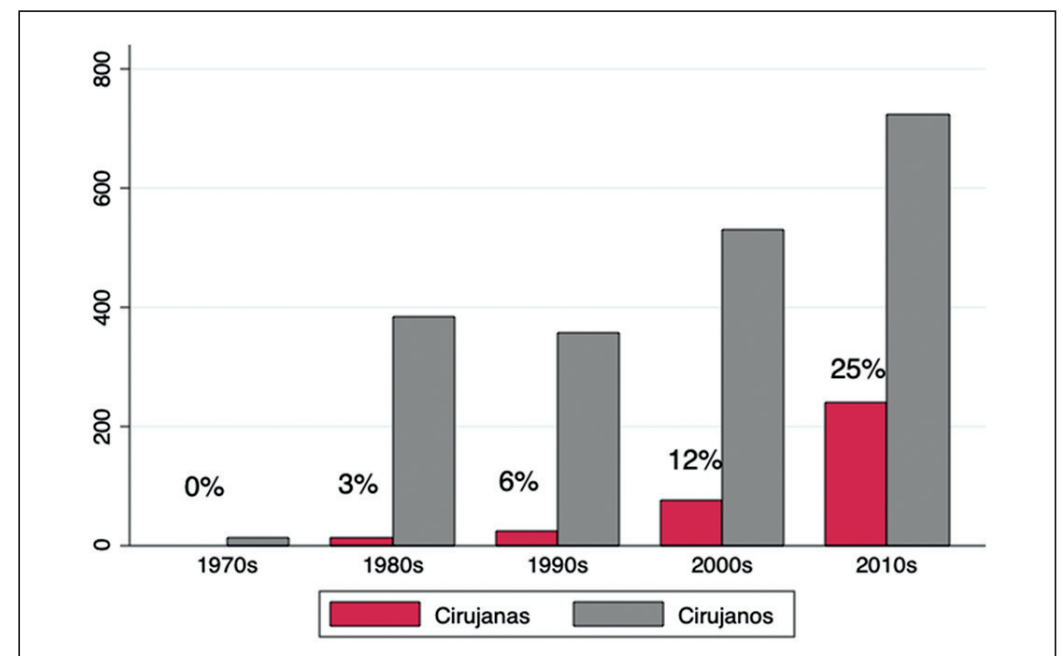

Figura 3. Evolución histórica del porcentaje de cirujanas egresadas por década en los últimos 50 años en Chile, certificadas por universidades o CONACEM. Fuente: Superintendencia de Salud. exclusivamente del registro de CONACEM, dado que la Superintendencia de Salud no registra cirugía digestiva como subespecialidad. La certificación por CONACEM no es automática ni obligatoria, por lo que las cifras pueden estar subvaloradas. La información acerca de la subespecialidad de cirugía de mama, fue obtenida del listado de socios activos de Sociedad de Mastología (excluyendo otras especialidades que no fueran cirugía, corroborado con datos de la Superintendencia), dado que CONACEM y Superintendencia de Salud no registran cirugía de mama como subespecialidad.

La edad, lugar de ejercicio de la profesión y pertenencia a la Sociedad de Cirujanos de Chile, fueron obtenidos a través de una encuesta online (Catastro Nacional) realizada por la Asociación de Cirujanas de Chile en los meses de abril y mayo de 2020. Esta es una red que agrupa a cirujanas de todo Chile y a residentes de género femenino (279 miembros a abril de 2020), cuyo fin es el promover la equidad de género en cirugía. El porcentaje de cirujanas en formación se obtuvo a través de recolección de datos de los 26 centros formadores de Cirugía en Chile.

\section{Resultados}

En los últimos 50 años, el porcentaje de mujeres egresadas de Escuelas de Medicina en Chile ha tenido un aumento importante, desde los años 70 en que correspondían a menos del $20 \%$ hasta el 2018 en que sobrepasaron el $50 \%$ de los egresados (Figura 1). Las mujeres corresponden al $43 \%$ del total de los médicos activos del país. Sin embargo, las mujeres en Cirugía sólo corresponden al 15\% del total (351 mujeres, 2009 hombres) (Figura 2).

El porcentaje de cirujanas certificadas se ha duplicado en cada década incluida en el estudio: $3 \%$ en la década de los $80,6 \%$ en los $90,12 \%$ en la década de 2000 , y $25 \%$ en la década del 2010 (Figura 3). Existe variabilidad en el porcentaje de cirujanas que han entrenado en los distintos centros formadores, con un rango entre $0 \%$ y $60 \%$. Programas más nuevos, como la Universidad Católica del Maule y la Universidad Católica del Norte, cuentan con $60 \%$ y $36 \%$ de egresadas de género femenino respectivamente, mientras que, en centros de más larga tradición en la formación quirúrgica como la Universidad de Chile, Universidad Católica y Universidad de Concepción, solo $14 \%$ de sus egresadas han sido mujeres (Tabla 1).

La Tabla 2 muestra la participación de las cirujanas en las diferentes subespecialidades. Las 
Tabla 1. Proporción de cirujanas egresadas por centro formador

\begin{tabular}{|lcccc|}
\hline Entidad acreditadora & Cirujanas & \% & Cirujanos & \% \\
\hline U. Católica del Maule & 3 & 60 & 2 & 40 \\
\hline U. Católica de Norte & 5 & 36 & 9 & 64 \\
\hline U. San Sebastián & 2 & 25 & 6 & 75 \\
U. del Desarrollo & 6 & 22 & 21 & 78 \\
\hline U. de Valparaíso & 43 & 21 & 158 & 79 \\
U. de los Andes & 9 & 20 & 35 & 80 \\
\hline U. de Santiago & 24 & 19 & 103 & 81 \\
U. de Chile & 99 & 14 & 593 & 86 \\
\hline U. Católica & 29 & 14 & 181 & 86 \\
U. de Concepción & 14 & 14 & 86 & 86 \\
CONACEM & 100 & 13 & 666 & 87 \\
\hline U. Austral & 9 & 12 & 66 & 88 \\
\hline U. de la Frontera & 7 & 11 & 54 & 89 \\
\hline U. Andrés Bello & 1 & 9 & 10 & 91 \\
\hline U. Finis Terrae & 0 & 0 & 4 & 100 \\
\hline U. Diego Portales & 0 & 0 & 10 & 100 \\
\hline
\end{tabular}

Fuente: Superintendencia de Salud.

cirujanas son una mayor proporción en cirugía de mama $(42,0 \%)$, cirugía plástica $(21,8 \%)$ y cirugía de cabeza y cuello (19,6\%). La especialidad en que hay menos proporción de mujeres es cirugía cardíaca, con $2,5 \%$.

En cuanto a la edad de las cirujanas, éstas son 10 años más jóvenes que sus pares masculinos, 41 años (rango 28-80 años) para las mujeres y 51 (rango 28-94 años) para los hombres. Las cirujanas están presentes en todo Chile, desde Arica a Punta Arenas, con excepción de Antofagasta y Copiapó. El $80 \%$ de las cirujanas se concentra en la zona central del país (Figura 4). La Figura 5 muestra la participación de las cirujanas en la Sociedad de Cirujanos de Chile: $3 \%$ es miembro y ha formado parte del directorio, $18 \%$ es miembro titular, $46 \%$ es miembro asociado y $33 \%$ no es miembro de la Sociedad.

El número de residentes de género femenino actualmente en formación es de 105 de un total de $314(33,4 \%)$, siendo el $36 \%$ en $1^{\text {er }}$ año, $33 \%$ en $2^{\circ}$ y $34 \%$ en $3^{\text {er }}$ año.
Tabla 2. Porcentaje de cirujanas certificadas por universidades o CONACEM en diferentes subespecialidades

\begin{tabular}{|lccc|}
\hline Subespecialidad & Mujeres & Hombres & \% \\
\hline Mama* & 22 & 31 & 42,0 \\
Plástica & 36 & 165 & 21,8 \\
Cabeza y Cuello & 11 & 56 & 19,6 \\
Vascular & 13 & 130 & 10,0 \\
Coloproctología & 8 & 91 & 8,8 \\
\hline Tórax & 6 & 71 & 8,5 \\
\hline Digestivo** & 4 & 52 & 7,7 \\
Cardiocirugía & 2 & 77 & 2,6 \\
\hline
\end{tabular}

Fuente: Superintendencia de Salud. *Información obtenida de listado de socios activos de Sociedad de Mastología, dado que CONACEM y Superintendencia de Salud no registran cirugía de mama como subespecialidad. **Información obtenida exclusivamente del registro de CONACEM, dado que Superintendencia de Salud no registra Cirugía Digestiva como subespecialidad. La certificación por CONACEM no es automática ni obligatoria, por lo que las cifras pueden estar subvaloradas. 


\section{ARTÍCULO ORIGINAL}

Figura 4. Distribución geográfica de las cirujanas en el territorio nacional (abril 2020).

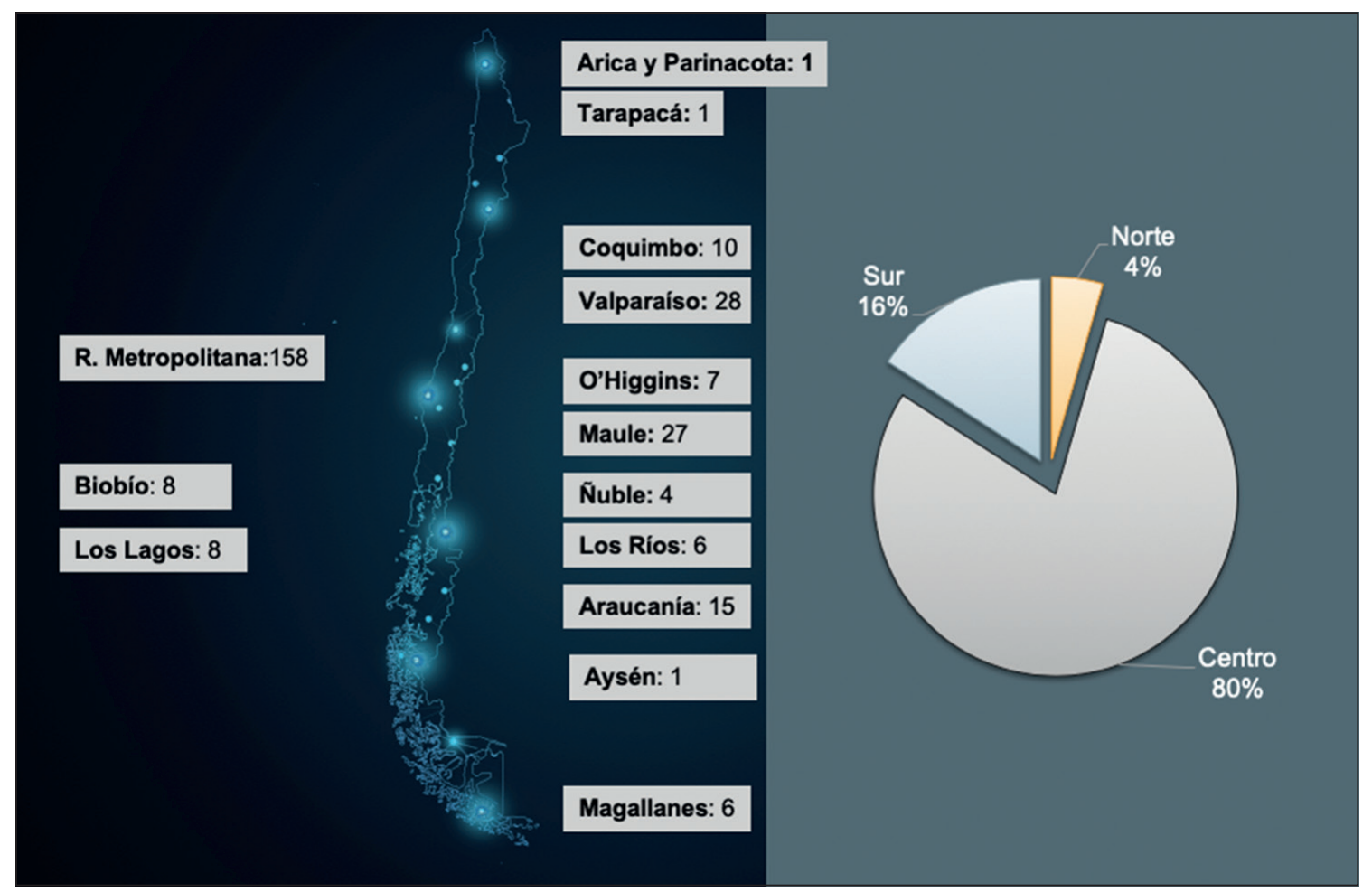

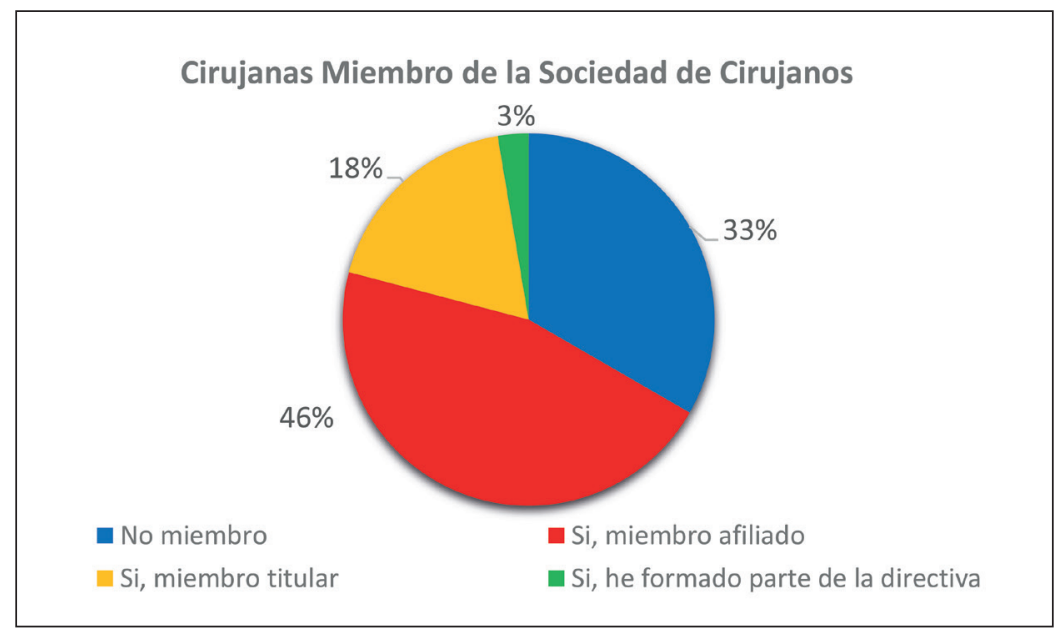

Figura 5. Participación de las cirujanas en la Sociedad de Cirujanos de Chile.

\section{Discusión}

Las mujeres se han incorporado a la actividad laboral en todas las áreas, y la cirugía no es una excepción. Si bien en los últimos cincuenta años, las mujeres egresadas de Medicina en Chile aumen- taron progresivamente hasta superar el $50 \%$ por año, este aumento no ha logrado traducirse aún en un aumento significativo en cirugía, donde las mujeres solo corresponden al 15\%. ¿Por qué un número tan bajo de mujeres en cirugía? ¿Existen barreras para las mujeres para entrar a cirugía? Múltiples estudios demuestran que las estudiantes de Medicina pueden verse desincentivadas de elegir una carrera quirúrgica por diferentes motivos: actitudes sexistas en sus rotaciones por cirugía e internado, escasez de modelos a seguir, dudas acerca del estilo de vida $^{7-9}$. La concepción antigua que la cirugía es una especialidad para hombres ha sido transmitida de generación en generación y aún se sigue entregando este mensaje a alumnas e internas. Existe evidencia que la exposición temprana a mentoras y modelos a seguir hace más probable que las estudiantes consideren una carrera quirúrgica ${ }^{10,11}$. Un estudio realizado en estudiantes de medicina en Colombia, demostró que la exposición temprana a modelos se asoció significativamente con la intención de optar por una carrera en cirugía ${ }^{12}$. Los modelos pueden influir en la consideración de la cirugía como opción de vida, permiten que los estudiantes puedan mejorar las percepciones sobre el estilo de vida y disipar estereotipos negativos ${ }^{13}$. Si solo $15 \%$ de los 
cirujanos en Chile son mujeres, es muy probable que muchas de las estudiantes no tengan exposición a modelos femeninos a imitar. Un número importante de residentes desearía mentores del mismo género, dado que además de orientación en opciones de carrera, muchos de los tópicos que desean discutir tienen que ver con balance vida personal y carrera ${ }^{14}$. La cirugía es una especialidad demandante, por lo que las mujeres pueden sentir que el desarrollo de una familia puede ser poco compatible con la especialidad $^{12}$. Para entender a cabalidad cuáles son las barreras para las mujeres en cirugía es necesario realizar estudios cualitativos en profundidad.

El número de mujeres certificadas como cirujanos ha aumentado progresivamente en los últimos cincuenta años, duplicándose en cada década, aumentando del $3 \%$ de los cirujanos en la década de los 80 hasta $25 \%$ en la década de 2010 . Es esperable que este número siga aumentando, ya que actualmente el número de residentes mujeres es de $33 \%$. Existe variabilidad respecto al porcentaje de mujeres que se forman en los diferentes centros, entre $0 \%$ y $60 \%$. Programas más nuevos como la Universidad Católica del Maule y la Universidad Católica del Norte parecen ser más "amigables" con las residentes mujeres, ya que cuentan con un mayor número de egresadas de género femenino (60\% y 36\% respectivamente). En uno de estos centros hubo una cirujana jefa de servicio por 10 años, lo que refuerza la importancia de las mujeres en posiciones de liderazgo para atraer a más mujeres en la especialidad. Es interesante que programas con trayectoria más larga como la Universidad de Chile, Universidad Católica y Universidad de Concepción, cuentan con solo $14 \%$ de mujeres entre sus egresados. Otros Centros no han tenido egresadas mujeres. Sería interesante conocer los factores que explican esta variabilidad, si existe algún tipo de sesgo que podría mejorarse, investigando qué buenas prácticas podrían ser adoptadas por los centros con menor representación femenina.

Datos del catastro de la Asociación de Cirujanas muestran que $35 \%$ de las cirujanas en Chile se desempeñan como cirujanas generales. Las cirujanas certificadas con subespecialidad se concentran principalmente en las áreas de cirugía de mama, cirugía plástica y cirugía de cabeza y cuello. Ha existido probablemente un sesgo histórico de aceptar a mujeres en "cirugía de superficie", y en la medida que estas cirujanas fueron desarrollándose en estas áreas, les fueron abriendo el camino a otras mujeres. Sin embargo, hoy existen cirujanas coloproctólogas, vasculares, torácicas, digestivas y cardiocirujanas. Las cirujanas están presentes en todo el territorio nacional, desde Arica a Punta Arenas, con excepción de algunas regiones, concentrándose el $80 \%$ en el centro del país al igual que la población general.

La participación de cirujanas en cargos directivos de la Sociedad de Cirujanos de Chile ha sido solamente de un $3 \%$, con una sola mujer presidenta en la historia de la Sociedad, la Dra. Jamile Camacho el año 2010. Llama la atención que 33\% de las cirujanas no pertenece a la Sociedad de Cirujanos, lo que probablemente puede deberse a un sentimiento de no representatividad (dato no estudiado). El nombre Sociedad de Cirujanos, puede contribuir a este sentimiento. La Real Academia Española establece que en las profesiones que tienen un sustantivo femenino, éste debe ser empleado obligatoriamente en caso de referirse a una mujer, en este caso, cirujanas. Una forma de promover una participación más activa de cirujanas en la Sociedad y en la especialidad puede ser aumentar el número de mujeres en la directiva, buscando activamente la elección de un número determinado de mujeres. Políticas públicas mundiales y nacionales que buscan lograr equidad de género han propuesto un sistema de cuotas, para poder nivelar a un grupo que ha estado en desventaja. Por ejemplo, el programa "mujeres protagonistas" del Ministerio de la Mujer propone que las mujeres formen parte del $40 \%$ de los directorios de las empresas e instituciones públicas ${ }^{15}$. Los reconocimientos académicos también han sido escasos, con solo una maestra de la cirugía, la Dra. Ivonne Pabst en el año 2000 , pese a que las mujeres han estado presentes en la cirugía desde mediados del siglo pasado ${ }^{1,16}$. Una estrategia para fomentar la participación de las cirujanas en la Sociedad de Cirujanos, podría ser formar un Departamento de Cirujanas, cuya función sea promover la actividad científica de sus miembros, fomentar la organización de instancias científicas y desarrollar un programa de mentoría.

Las limitaciones de este estudio es que las estadísticas se basan principalmente en los registros de la Superintendencia de Salud, por lo que cualquier inexactitud en la recolección de estos datos pudiera afectar los resultados de este estudio. Algunas subespecialidades no están certificadas por CONACEM (cirugía de mama) o han sido certificadas recientemente (cirugía digestiva), por lo que los porcentajes de participación de las cirujanas en estas especialidades fueron estimados usando registros de la Sociedad de Mastología y registro de CONACEM, que pudieran no ser exactos por lo que solo sirven como aproximación.

En conclusión, las mujeres han contribuido a la cirugía del país desde hace más de 50 años. Actualmente las cirujanas representan solo el 15\% 
de la fuerza quirúrgica; sin embargo, este número ha aumentado progresivamente duplicándose en cada década y se espera seguirá aumentando con un $33 \%$ de los residentes de cirugía actuales de género femenino. Se requiere más cirujanas en cargos de representación y liderazgo que sirvan como modelos para las nuevas generaciones para lograr mayor participación femenina en la especialidad.

\section{Agradecimientos}

A Andrea Quinchavalí por su ayuda en la recolección de los datos de los centros formadores.

\section{Responsabilidades éticas}

Protección de personas y animales. Los autores declaran que para esta investigación no se han realizado experimentos en seres humanos ni en animales.

Confidencialidad de los datos. Los autores declaran que en este artículo no aparecen datos de pacientes.

Conflictos de interés: no hay.

\section{Bibliografía}

1. Valenzuela M. La mujer cirujano: ¿Todavía un "cuento para niñas rebeldes"? Rev Cir. 2020;72:7-8.

2. Wirtzfeld DA. The history of women in surgery. Can J Surg. 2009;52:317-20.

3. Espinoza R, Onetto C, Lombardi J. La incorporación de la mujer en la cirugía chilena. Rev Chil Cir. 2012;64:487-91.

4. Instituto Nacional de Estadísticas.

Género y Mercado Laboral. https:// wwwinecl/docs/default-source/ genero/infograf $\% \mathrm{C} 3 \% \mathrm{ADas} /$ infograf $\% \mathrm{C} 3 \% \mathrm{ADas} 2 /$ infograf $\% \mathrm{C} 3 \% \mathrm{ADa}$-géneroy-mercado-laboral-(ene)2020pdf?sfvrsn=500dbfe5 3. 2020; Consultado el 24 de julio de 2020.

5. Instituto Nacional de Estadísticas. Educación y Género. https:// www.ine.cl/docs/default-source/ genero/infograf $\% \mathrm{C} 3 \% \mathrm{ADas} /$ infograf $\% \mathrm{C} 3 \% \mathrm{ADas} 2 /$ infograf $\% \mathrm{C} 3 \% \mathrm{ADa}$-educación-y-género(sies)-2020pdf?sfvrsn=d56ce5b_3. 2020; Consultado el 24 de julio de 2020.

6. http://www.supersalud.gob.cl/ difusion/665/w3-propertyvalue-4018. html. Registro Nacional de prestadores de Salud. Consultado 12 de junio de 2020.

7. Bernardi K, Shah P, Lyons NB, Olavarría OA, Alawadi ZM, Leal IM, et al. Perceptions on gender disparity in surgery and surgical leadership: A multicenter mixed methods study. Surgery 2020;167:743-50.

8. Burgos CM, Josephson A. Gender differences in the learning and teaching of surgery: a literature review. Int J Med Educ. 2014;5:110-24.

9. Myers SP, Hill KA, Nicholson KJ, Neal MD, Hamm ME, Switzer GE, et al. A qualitative study of gender differences in the experiences of general surgery trainees. J Surg Res. 2018;228:127-34

10. Myers SP, Littleton EB, Hill KA, Dasari M, Nicholson KJ, Knab LM, et al. Perceptions Regarding Mentorship Among General Surgery Trainees With Academic Career Intentions. J Surg Educ. 2019;76:916-23.

11. Faucett EA, McCrary HC, Milinic T, Hassanzadeh T, Roward SG, Neumayer LA. The role of same-sex mentorship and organizational support in encouraging women to pursue surgery. Am J Surg. 2017;214:640-4.

12. Domínguez LC, Sierra D, Vega V, Gómez L, Pacheco M, Vargas F. ¿Tienes lo que hay que tener?: un análisis de los factores asociados con la intención de optar por una carrera en cirugía entre estudiantes de medicina. Rev Cir. 2020;72:22-9.

13. Sinclair P, Fitzgerald JE, Hornby ST, Shalhoub J. Mentorship in surgical training: current status and a needs assessment for future mentoring programs in surgery. World J Surg. 2015;39:303-13; discussion 14.

14. Luc JGY, Stamp NL, Antonoff MB. Social media in the mentorship and networking of physicians: Important role for women in surgical specialties. Am J Surg. 2018;215:752-60.

15. Ministerio de la Mujer. Mujeres Protagonistas. https://minmujeryeggob. cl/?page_id=4195. 2020; Consultado el 24 de julio de 2020.

16. Sepúlveda S. Las mujeres en la medicina chilena: Desde los tiempos de Eloísa Díaz a la actualidad. Rev Med Chile 2019;147:1184-9. 